\title{
Two Mechanisms of Packet Dropout Compensation for Linear Filter of Networked Control Systems
}

\author{
Hao $\operatorname{Jin}^{1}$ and Jing $\mathrm{Ma}^{2 *}$ \\ ${ }^{1}$ School of Computer and Information Engineering, Harbin University of \\ Commerce, Harbin 150028, China \\ 2 School of Mathematics Science, Heilongjiang University, Harbin 150080, China \\ jingma427@163.com
}

\begin{abstract}
This paper studies the optimal linear filtering problem for networked control systems (NCSs) with multiple packet dropouts. When the current measurement is lost, the two popular compensation mechanisms (the hold-input and zero-input mechanism) are used for compensation, respectively. The hold-input strategy means that the latest measurements received is applied directly whereas the zero-input one adopts zero value. Based on the two popular compensators, the optimal linear filters in the linear minimum variance sense are given by innovation analysis approach, and their performances are compared in terms of two simulation examples. The conclusion is that neither of the two compensation mechanisms can be claimed to be superior to the other.
\end{abstract}

Keywords: Packet dropout; Compensation; Linear filter; Networked control system; Innovation analysis approach

\section{Introduction}

Recently, the control and filtering problem for NCSs have received much attention due to their wide applications in target tracking, environmental monitoring, and communication [1-4]. Because of the communication noise, interference or congestion, random packet dropouts could occur in data transmissions, which is challenging for filtering of the system.

For systems with packet dropouts, from the literatures, there are two popular compensation mechanisms: the hold-input and the zero-input mechanisms. The former means that the latest measurement or control signal received is used whereas the latter adopts zero value whenever the current signal is lost. The two compensators are straight forward and easy to implement. Based on the hold-input compensator, the stabilization problem of networked controls by a switched system approach [5], the optimal $\mathrm{H}_{2}$ filtering problem by LIM approach [6], the optimal linear estimation and steady-state estimation problem by innovation analysis approach [7-8] are investigated, respectively. Based on the zero-input compensator, the optimal filtering problem for systems with multi-step random delays and multiple packet dropouts are studied in [9]. Further, the possible packet dropouts for a rehabilitation system [10] and for T-S fuzzy dynamic systems [11] are tackled, respectively. Using the two compensators, the optimal control problem of LTI system was investigated in [12], the LG performance are analyzed in [13], and the LQG problem is investigated in [14]. Moreover, a one-step prediction-based packet dropout compensation method is proposed and the corresponding control problem is also studied in [15] and [16].

${ }^{*}$ Corresponding Author: Jing Ma 
Motivated by the above discussion, in this paper, we investigate the optimal linear filtering problem for networked systems with multiple packet dropouts by applying the two popular compensators. First, we review the existing linear filter proposed in [7] based on the hold-input mechanism. However, the proposed filter in [7] has high computational cost due to the state augmentation. Then, we propose the optimal filter by innovation analysis approach based on the zero-input mechanism. The proposed filter has the same order with the original system and can reduce the computational burden. Finally, we use two examples to compare the filtering performance of the above two filters. The results show that neither of the two compensators always outperforms the other.

\section{Problem Formulation}

Consider the following linear discrete-time system modeled by

$$
\begin{array}{r}
x(t+1)=\Phi x(t)+\Gamma w(t) \\
z(t)=H x(t)+v(t)
\end{array}
$$

where $x(t) \in \square^{n}$ is the state, $z(t) \in \square^{m}$ is the sensor output. $\Phi, \Gamma$ and $H$ are constant matrices. $w(t) \in \square^{r}$ and $v(t) \in \square^{m}$ are correlated zero-mean white noises with covariance matrices $\mathrm{E}\left[w(t) w^{\mathrm{T}}(t)\right]=Q_{w}, \mathrm{E}\left[v(t) v^{\mathrm{T}}(t)\right]=Q_{v}$ and $\mathrm{E}\left[w(t) v^{\mathrm{T}}(t)\right]=S$. The initial state $x(0)$ is independent of $w(t)$ and $v(t)$, and has $\mathrm{E}\{x(0)\}=\sigma_{0}$ and $\mathrm{E}\left\{\left(x(0)-\sigma_{0}\right)\left(x(0)-\sigma_{0}\right)^{\mathrm{T}}\right\}=\Sigma_{0}$.

We assume that there exist possible consecutive packet dropouts during the data transmission from the sensor to the filter over a network. Then, a compensation measurement is needed at the filter when the current measurement is not available.

Now, we review the two popular compensation mechanisms:

$$
\begin{aligned}
& \text { Hold-input mechanism: } y_{h}(t)=\eta(t) z(t)+(1-\eta(t)) y_{h}(t-1) \\
& \text { Zero-input mechanism: } y_{z}(t)=\eta(t) z(t)
\end{aligned}
$$

where $\eta(t)$ is an i.i.d Bernoulli process with $P\{\eta(t)=1\}=\bar{\eta}$ and $P\{\eta(t)=0\}=1-\bar{\eta}$. The subscripts $\mathrm{z}$ and $\mathrm{h}$ in the compensation measurements indicate the zero-input and the holdinput mechanism, respectively.

Remark 1: From (3) and (4), we see that if $\eta(t)=0$, i.e., the current measurement is lost, the latest measurement is used in the hold-input mechanism whereas zero is used in the zero-input mechanism.

\section{Optimal Linear Filters under the Two Compensation Mechanisms}

In this section, we will give the optimal linear filters based on the above two compensation mechanisms.

\subsection{Linear Filter under the Hold-input Compensator}

In this subsection, we shall review the filter under the hold-input mechanism [7]. First, we rewrite the system (1)-(3) as the following form of [7]:

$$
\begin{gathered}
X(t+1)=\left[\begin{array}{cc}
\Phi & 0 \\
\eta(t) H & (1-\eta(t)) I_{m}
\end{array}\right] X(t)+\left[\begin{array}{cc}
\Gamma & 0 \\
0 & \eta(t) I_{m}
\end{array}\right] W(t) \\
y_{h}(t)=\left[\begin{array}{ll}
\eta(t) H & (1-\eta(t)) I_{m}
\end{array}\right] x(t)+\eta(t) v(t)
\end{gathered}
$$

where $X(t)=\left[\begin{array}{ll}x^{\mathrm{T}}(t) & y_{h}^{\mathrm{T}}(t)\end{array}\right]^{\mathrm{T}}, W(t)=\left[\begin{array}{ll}w^{\mathrm{T}}(t) & v^{\mathrm{T}}(t)\end{array}\right]^{\mathrm{T}}$.

Lemma 1 [7] For systems (5)-(6), the optimal linear filter is given by

$$
\begin{gathered}
\hat{X}_{h}(t \mid t)=\hat{X}_{h}(t \mid t-1)+K_{h}(t) \varepsilon_{h}(t) \\
\hat{X}_{h}(t+1 \mid t)=\bar{\Phi} \hat{X}_{h}(t \mid t-1)+L_{h}(t) \varepsilon_{h}(t) \\
\varepsilon_{h}(t)=y_{h}(t)-\bar{H} \hat{X}_{h}(t \mid t-1) \\
K_{h}(t)=P_{h}(t \mid t-1) \bar{H}^{\mathrm{T}} Q_{\delta_{h}}^{-1}(t)
\end{gathered}
$$




$$
\begin{gathered}
L_{h}(t)=\left\{\bar{\eta}(1-\bar{\eta})\left[\begin{array}{cc}
0 & 0 \\
H & -I_{m}
\end{array}\right] q_{X}(t)\left[H \quad-I_{m}\right]^{\mathrm{T}}+\bar{\Phi} P(t \mid t-1) \bar{H}^{\mathrm{T}}+\bar{\eta}\left[\begin{array}{c}
\Gamma S \\
Q_{v}
\end{array}\right]\right\} Q_{\varepsilon_{h}}^{-1}(t) \\
Q_{\varepsilon_{h}}(t)=\bar{\eta}(1-\bar{\eta})\left[\begin{array}{ll}
H & -I_{m}
\end{array}\right] q_{X}(t)\left[\begin{array}{ll}
H & -I_{m}
\end{array}\right]^{\mathrm{T}}+\bar{\eta} Q_{v}+\bar{H} P(t \mid t-1) \bar{H}^{\mathrm{T}} \\
q_{X}(t+1)=\bar{\Phi} q_{X}(t) \bar{\Phi}^{\mathrm{T}}+\bar{\eta}(1-\bar{\eta})\left[\begin{array}{cc}
0 & 0 \\
H & -I_{m}
\end{array}\right] q_{X}(t)\left[\begin{array}{cc}
0 & 0 \\
H & -I_{m}
\end{array}\right]^{\mathrm{T}}+Q \\
P_{h}(t \mid t)=P_{h}(t \mid t-1)-K_{h}(t) Q_{\varepsilon_{h}}(t) K_{h}^{\mathrm{T}}(t) \\
\left.P_{h}(t+1 \mid t)=\bar{\eta}(1-\bar{\eta})\left\{\left[\begin{array}{cc}
0 & 0 \\
H & -I_{m}
\end{array}\right]-L_{h}(t)\left[H \quad-I_{m}\right]\right\}\right\} q_{X}(t)\left\{\left[\begin{array}{cc}
0 & 0 \\
H & -I_{m}
\end{array}\right]-L_{h}(t)\left[H \quad-I_{m}\right]\right\}^{\mathrm{T}}+Q \\
+\left(\bar{\Phi}-L_{h}(t) \bar{H}\right) P_{h}(t \mid t-1)\left(\bar{\Phi}-L_{h}(t) \bar{H}\right)^{\mathrm{T}}-\bar{\eta} L_{h}(t)\left[\begin{array}{c}
\Gamma S \\
Q_{v}
\end{array}\right]^{\mathrm{T}}-\bar{\eta}\left[\begin{array}{c}
\Gamma S \\
Q_{v}
\end{array}\right] L_{h}^{\mathrm{T}}(t)+\bar{\eta} L_{h}(t) Q_{v} L_{h}^{\mathrm{T}}(t)
\end{gathered}
$$

where $\bar{H}=\left[\begin{array}{ll}\bar{\eta} H & (1-\bar{\eta}) I_{m}\end{array}\right], \quad \bar{\Phi}=\left[\begin{array}{cc}\Phi & 0 \\ \bar{\eta} H & (1-\bar{\eta}) I_{m}\end{array}\right], Q=\left[\begin{array}{cc}\Gamma Q_{w} \Gamma^{\mathrm{T}} & \bar{\eta} \Gamma S \\ \bar{\eta} S^{\mathrm{T}} \Gamma^{\mathrm{T}} & \bar{\eta} Q_{v}\end{array}\right], \varepsilon_{h}(t)$ is the innovation sequence with variance $Q_{\varepsilon_{h}}(t), K_{h}(t)$ and $L_{h}(t)$ are gain matrices, $q_{X}(t)$ is the state second-order moment matrix of augmented state $X(t), P_{h}(t \mid t)$ is the filtering error variance matrix, $P_{h}(t \mid t-1)$ is the one-step prediction error variance matrix. The initial values are $\hat{X}_{h}(0 \mid-1)=\left[\begin{array}{ll}\sigma_{0} & 0\end{array}\right]^{\mathrm{T}}, P_{h}(0 \mid-1)=\operatorname{diag}\left(\Sigma_{0}, 0\right)$ and $q_{X}(0)=\operatorname{diag}\left(\sigma_{0} \sigma_{0}^{\mathrm{T}}+\Sigma_{0}, 0\right)$.

Remark 2: From the definition of $X(t)=\left[\begin{array}{ll}x^{\mathrm{T}}(t) & y_{h}^{\mathrm{T}}(t)\end{array}\right]^{\mathrm{T}}$, the linear filter under the hold-input mechanism can be given by $\hat{x}_{h}(t \mid t)=\left[\begin{array}{ll}I_{n} & 0\end{array}\right] \hat{X}_{h}(t \mid t)$.

\subsection{Linear Filter under the Zero-input Compensator}

In this subsection, we shall derive the linear filter under the zero-input mechanism based on the innovation analysis approach.

Theorem 1. For systems (1), (2) and (4), the optimal linear filter is given by

$$
\begin{gathered}
\hat{x}_{z}(t \mid t)=\hat{x}_{z}(t \mid t-1)+K_{z}(t) \varepsilon_{z}(t) \\
\hat{x}_{z}(t+1 \mid t)=\Phi \hat{x}_{z}(t \mid t-1)+L_{z}(t) \varepsilon_{z}(t) \\
\varepsilon_{z}(t)=y_{z}(t)-\bar{\eta} H \hat{x}_{z}(t \mid t-1) \\
K_{z}(t)=\bar{\eta} P_{z}(t \mid t-1) H^{\mathrm{T}} Q_{\varepsilon_{z}}^{-1}(t) \\
L_{z}(t)=\bar{\eta}\left(\Phi P_{z}(t \mid t-1) H^{\mathrm{T}}+\Gamma S\right) Q_{\varepsilon_{z}}^{-1}(t) \\
Q_{\varepsilon_{z}}(t)=\bar{\eta}(1-\bar{\eta}) H q_{x}(t) H^{\mathrm{T}}+\bar{\eta} Q_{v}+\bar{\eta}^{2} H P_{z}(t \mid t-1) H^{\mathrm{T}} \\
q_{x}(t+1)=\Phi q_{x}(t) \Phi^{\mathrm{T}}+\Gamma Q_{w} \Gamma^{\mathrm{T}} \\
P_{z}(t \mid t)=P_{z}(t \mid t-1)-K_{z}(t) Q_{\varepsilon_{z}}(t) K_{z}^{\mathrm{T}}(t) \\
P_{z}(t+1 \mid t)=\Phi P_{z}(t \mid t-1) \Phi^{\mathrm{T}}+\Gamma Q_{w} \Gamma^{\mathrm{T}}-L_{z}(t) Q_{\varepsilon_{z}}(t) L_{z}^{\mathrm{T}}(t)
\end{gathered}
$$

where $\varepsilon_{z}(t), Q_{\varepsilon_{z}}(t), K_{z}(t), L_{z}(t), P_{z}(t \mid t)$ and $P_{z}(t \mid t-1)$ are of the similar definitions as the one in Lemma 1. $q_{x}(t)$ is the state second-order moment matrix of the original state $x(t)$. The initial values are $\hat{x}_{z}(0 \mid-1)=\sigma_{0}, P_{z}(0 \mid-1)=\Sigma_{0}$ and $q_{x}(0)=\sigma_{0} \sigma_{0}^{\mathrm{T}}+\Sigma_{0}$.

Proof: By projection, we have (16)-(18). The gain matrices $K_{z}(t)$ and $L_{z}(t)$ are defined as:

$$
K_{z}(t)=\mathrm{E}\left[x(t) \varepsilon_{z}^{\mathrm{T}}(t)\right] Q_{\varepsilon_{z}}^{-1}(t), L_{z}(t)=\mathrm{E}\left[x(t+1) \varepsilon_{z}^{\mathrm{T}}(t)\right] Q_{\varepsilon_{z}}^{-1}(t)
$$

Substituting (4) into (18), $\varepsilon_{z}(t)$ can be rewrite as

$$
\varepsilon_{z}(t)=[\eta(t)-\bar{\eta}] H x(t)+\bar{\eta} H \tilde{x}_{z}(t \mid t-1)+\eta(t) v(t)
$$


where the estimation error is $\tilde{x}_{z}(t \mid t-1)=x(t)-\hat{x}_{z}(t \mid t-1)$. From (26), and using $\mathrm{E}[\eta(t)-\bar{\eta}]=0, \quad x(t) \perp v(t), \quad \tilde{x}_{z}(t \mid t-1) \perp \hat{x}_{z}(t \mid t-1) \quad, \quad \tilde{x}_{z}(t \mid t-1) \perp v(t), \quad$ and $\tilde{x}_{z}(t \mid t-1) \perp w(t)$, where the symbol " $\perp$ "denotes orthogonality, we have

$$
\begin{gathered}
\mathrm{E}\left[x(t) \varepsilon_{z}^{\mathrm{T}}(t)\right]=\bar{\eta} \mathrm{E}\left[\tilde{x}_{z}(t \mid t-1) \tilde{x}_{z}^{\mathrm{T}}(t \mid t-1)\right] H^{\mathrm{T}}=\bar{\eta} P_{z}(t \mid t-1) H^{\mathrm{T}} \\
\mathrm{E}\left[x(t+1) \varepsilon_{z}^{\mathrm{T}}(t)\right]=\Phi \mathrm{E}\left[x(t) \varepsilon_{z}^{\mathrm{T}}(t)\right]+\bar{\eta} \Gamma S
\end{gathered}
$$

Substituting (27) into (25) yields (19) and (20). By substituting (26) into $Q_{\varepsilon_{z}}(t)=\mathrm{E}\left[\varepsilon_{z}(t) \varepsilon_{z}^{\mathrm{T}}(t)\right]$ and using $\mathrm{E}\left[(\eta(t)-\bar{\eta})^{2}\right]=\bar{\eta}(1-\bar{\eta})$, we obtain (21). From the state equation (1), we easily obtain the state second-order moment matrix $q_{x}(t)=\mathrm{E}\left[x(t) x^{\mathrm{T}}(t)\right]$ of state $x(t)$.

Next, we derive the estimation error variance matrices $P_{z}(t \mid t)=\mathrm{E}\left[\tilde{x}_{z}(t \mid t) \tilde{x}_{z}^{\mathrm{T}}(t \mid t)\right]$ and $P_{z}(t+1 \mid t)=\mathrm{E}\left[\tilde{x}_{z}(t+1 \mid t) \tilde{x}_{z}^{\mathrm{T}}(t+1 \mid t)\right]$. By subtracting (16) and (17) from $x(t)$ and $x(t+1)$ yields the error equations

$$
\begin{gathered}
\tilde{x}_{z}(t \mid t)=x(t)-\hat{x}_{z}(t \mid t)=\tilde{x}_{z}(t \mid t-1)-K_{z}(t) \varepsilon_{z}(t), \\
\tilde{x}_{z}(t+1 \mid t)=x(t+1)-\hat{x}_{z}(t+1 \mid t)=\Phi x(t)+\Gamma w(t)-\hat{x}_{z}(t+1 \mid t)=\Phi \tilde{x}_{z}(t \mid t-1)+\Gamma w(t)-L_{z}(t) \varepsilon_{z}(t)
\end{gathered}
$$

Rewrite (28) as

$$
\begin{gathered}
\tilde{x}_{z}(t \mid t)+K_{z}(t) \varepsilon_{z}(t)=\tilde{x}_{z}(t \mid t-1), \\
\tilde{x}_{z}(t+1 \mid t)+L_{z}(t) \varepsilon_{z}(t)=\Phi \tilde{x}_{z}(t \mid t-1)+\Gamma w(t)
\end{gathered}
$$

Using $\tilde{x}_{z}(t \mid t) \perp \varepsilon_{z}(t)$ and $\tilde{x}_{z}(t+1 \mid t) \perp \varepsilon_{z}(t)$, we have

$$
\begin{gathered}
P_{z}(t \mid t)-K_{z}(t) Q_{\varepsilon_{z}}(t) K_{z}^{\mathrm{T}}(t)=P_{z}(t \mid t-1), \\
P_{z}(t+1 \mid t)-L_{z}(t) Q_{\varepsilon_{z}}(t) L_{z}^{\mathrm{T}}(t)=\Phi P_{z}(t \mid t-1) \Phi^{\mathrm{T}}+\Gamma Q_{w} \Gamma^{\mathrm{T}}
\end{gathered}
$$

From (30), we obtain (23) and (24).

Remark 3. From Theorem 1, the computational cost of the filter under the zero-input mechanism has the order of magnitude $O\left((n)^{3}\right)$. Compared with the filter under the holdinput mechanism in Lemma 1 with the magnitude $O\left((n+m)^{3}\right)$, the computational cost can be reduced.

\section{Simulation Examples}

In this section, we give two examples to compare the filtering performance under the two compensation mechanisms.

Example 1: Consider the numerical example in [7].

$$
\begin{aligned}
& x(t+1)=\left[\begin{array}{cc}
1.7240 & -0.7788 \\
1 & 0
\end{array}\right] x(t)+\left[\begin{array}{l}
1 \\
0
\end{array}\right] w(t) \\
& z(t)=\left[\begin{array}{cc}
0.0286 & 0.0264 \\
1 & 1
\end{array}\right] x(t)+0.2 w(t)
\end{aligned}
$$

where $w(t)$ is white noise with mean 0 and variance 1 .

The tracking performance of the two filters under the two compensators with $\bar{\eta}=0.5$ as shown in Figure 1-2. The corresponding filtering error variances are shown in Fig 3. From Figure 1-3, we see that the filter under hold-input compensator has the better accuracy than the one under zero-input compensator. 

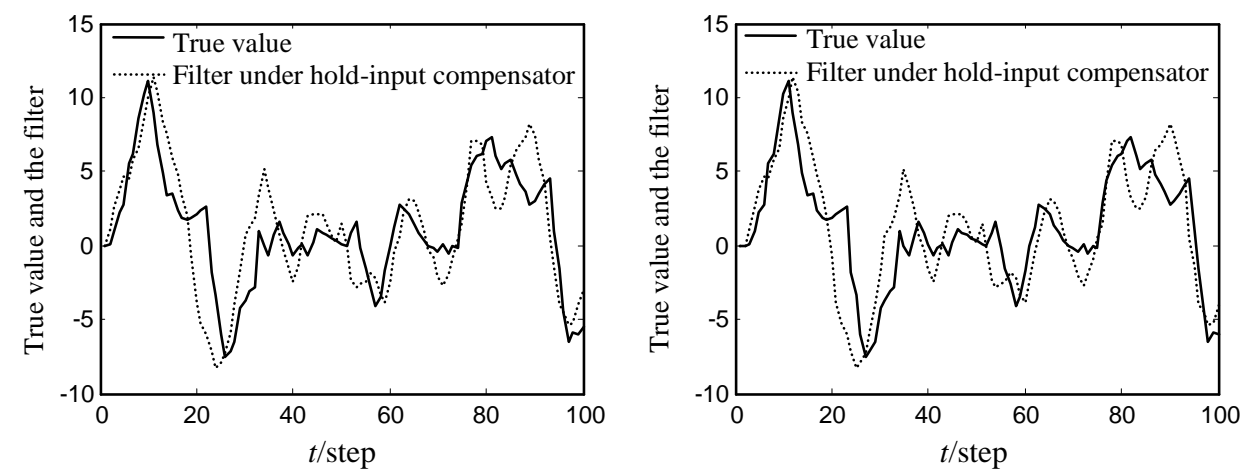

Figure 1. The Linear Filter under Hold-input Mechanism with $\bar{\eta}=0.5$
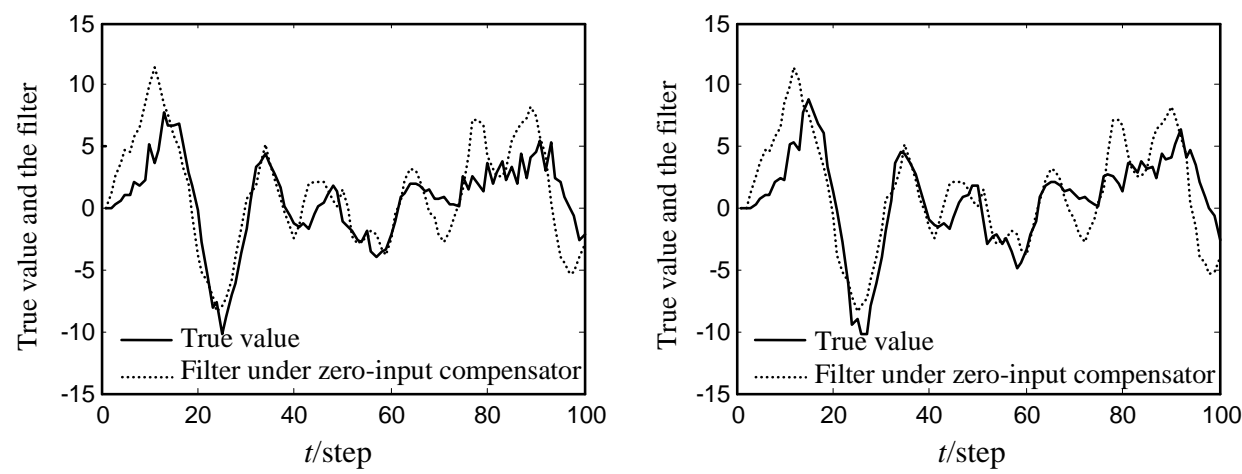

Figure 2. The Linear Filter under Zero-input Mechanism with $\bar{\eta}=0.5$
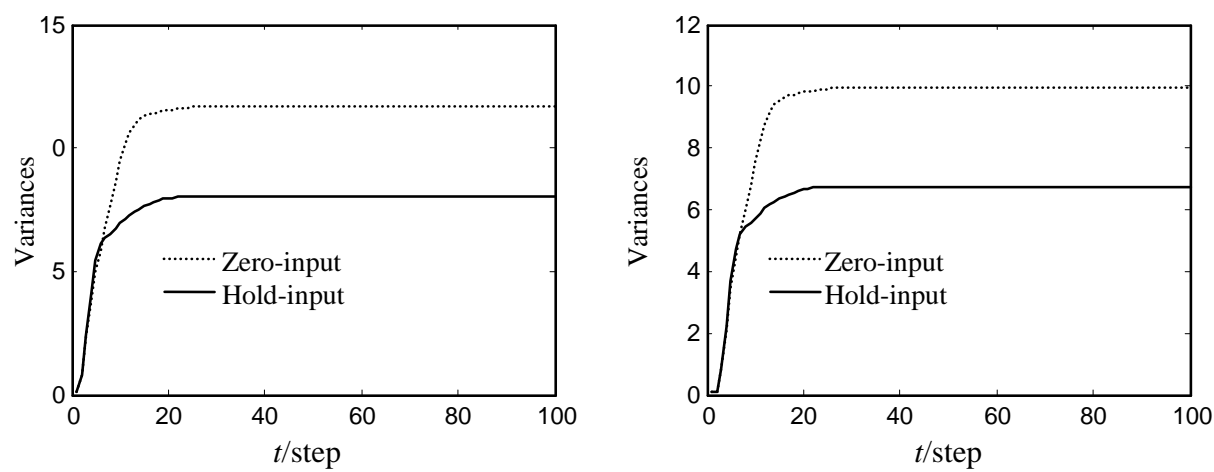

Figure 3. Filtering Error variances under the Two Compensation Mechanisms with $\bar{\eta}=0.5$

To further compare the performance of the two filters, the filtering error variances with a low arrival rate $\bar{\eta}=0.1$ are shown in Figure 4 . It can be seen that the zero-input mechanism performs better than the hold-input mechanism. The steady-state values of the filtering error variances of the two mechanisms with respect to different arrival rates $0.1 \leq \bar{\eta} \leq 1$ are shown in Figure 5. It can be concluded that neither of the two compensators always outperforms the other. 

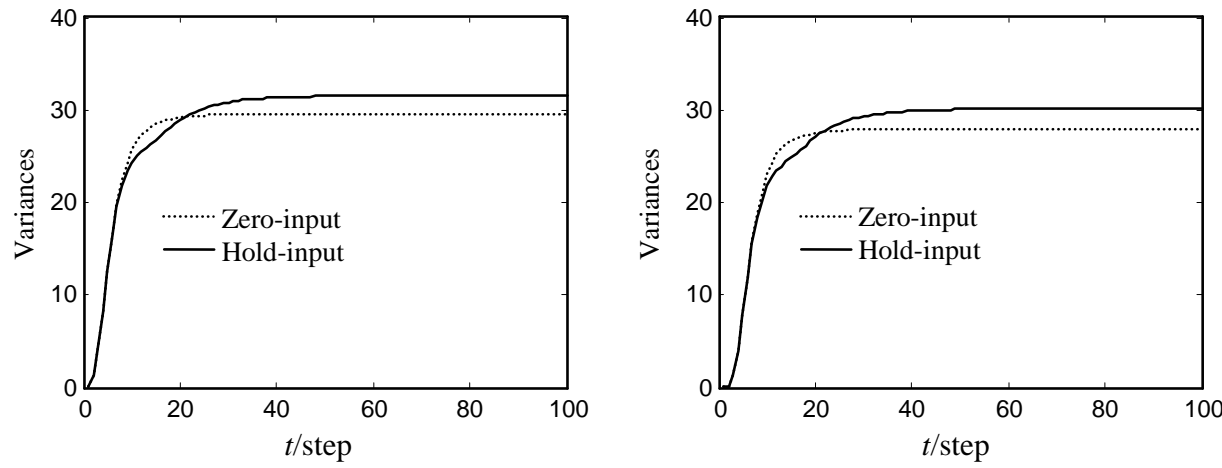

Figure 4. Filtering Error Variances under the Two Compensation Mechanisms with $\bar{\eta}=0.1$
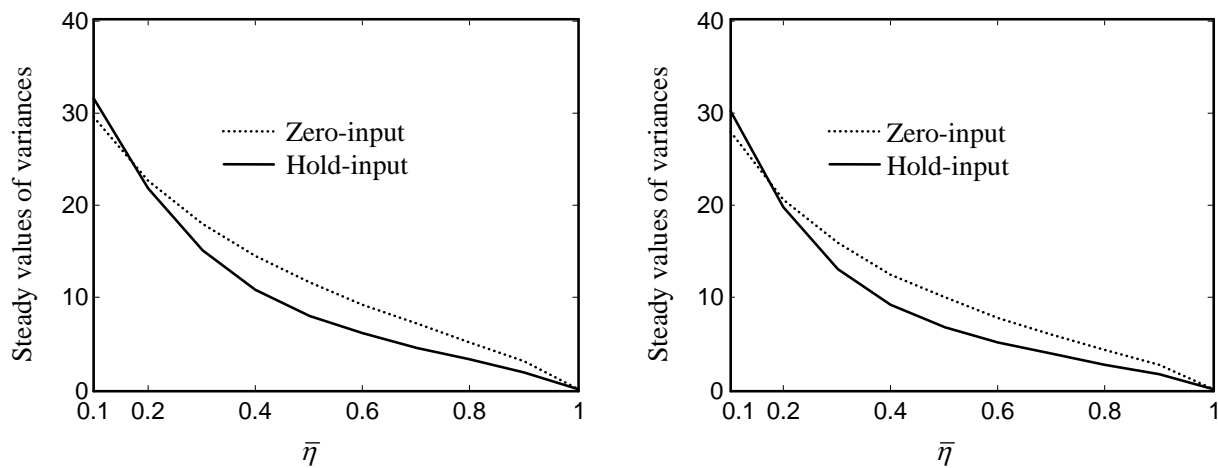

Figure 5. Filtering Error Variances under the two Compensation Mechanisms with different Arrival Rates $0.1 \leq \bar{\eta} \leq 1$

Example 2: Consider an uninterruptible power system (UPS) with $1 \mathrm{KVA}$ as in [17]. The corresponding direct-time parameter matrices are given as follows and all other variables are the same as example 1 .

$$
\Phi=\left[\begin{array}{ccc}
0.9226 & -0.6330 & 0 \\
1 & 0 & 0 \\
0 & 1 & 0
\end{array}\right], \quad \Gamma=\left[\begin{array}{c}
0.5 \\
0 \\
0.2
\end{array}\right], \quad H=\left[\begin{array}{lll}
23.738 & 20.287 & 0
\end{array}\right]
$$

The comparison curves under the two compensators are shown in Figure 7-8. It also can be concluded from Figure 7-8 that neither of the two compensation mechanisms can be claimed to be superior to the other. 

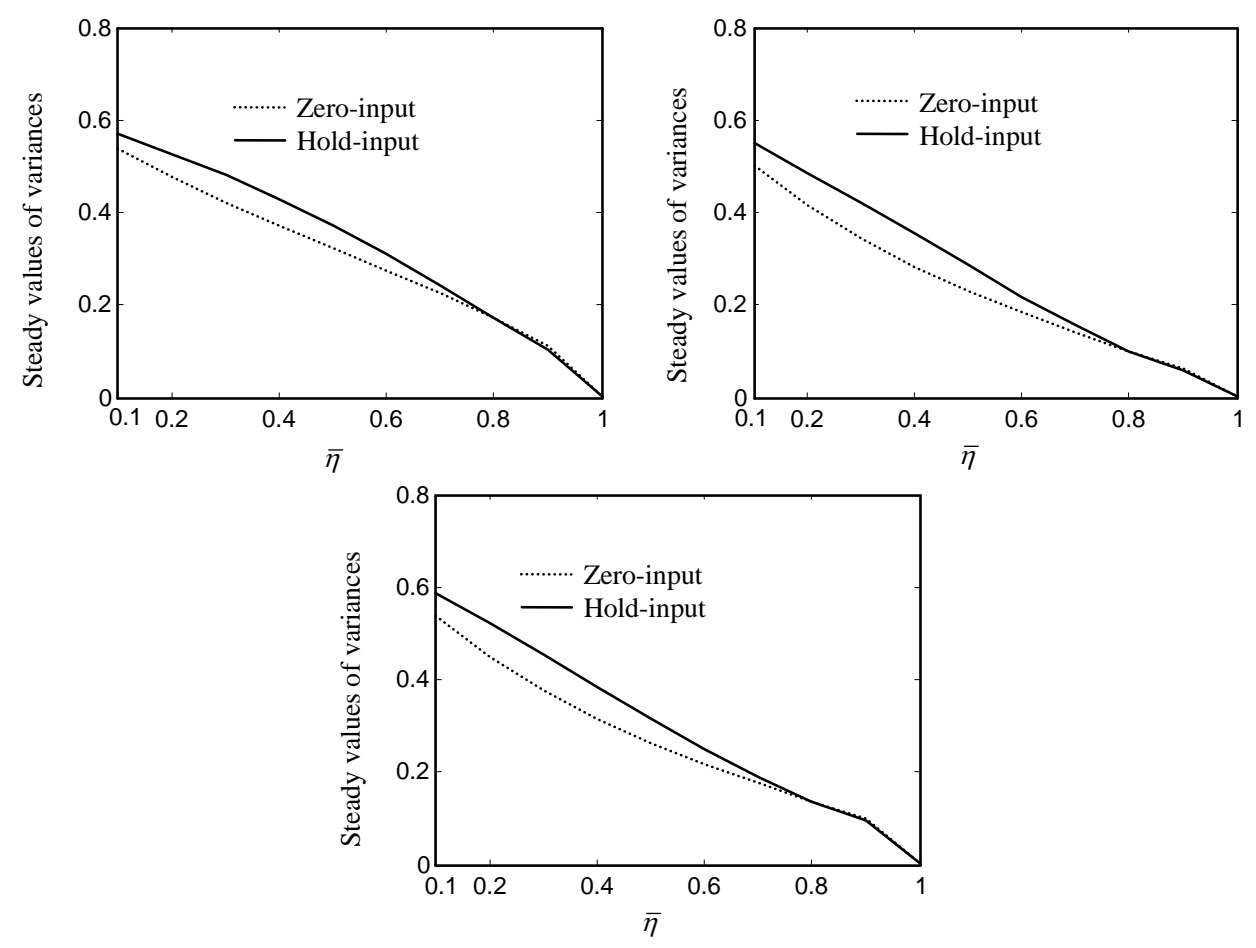

Figure 7. Filtering Error Variances under the Two Compensation Mechanisms with different Arrival Rates $0.1 \leq \bar{\eta} \leq 1$
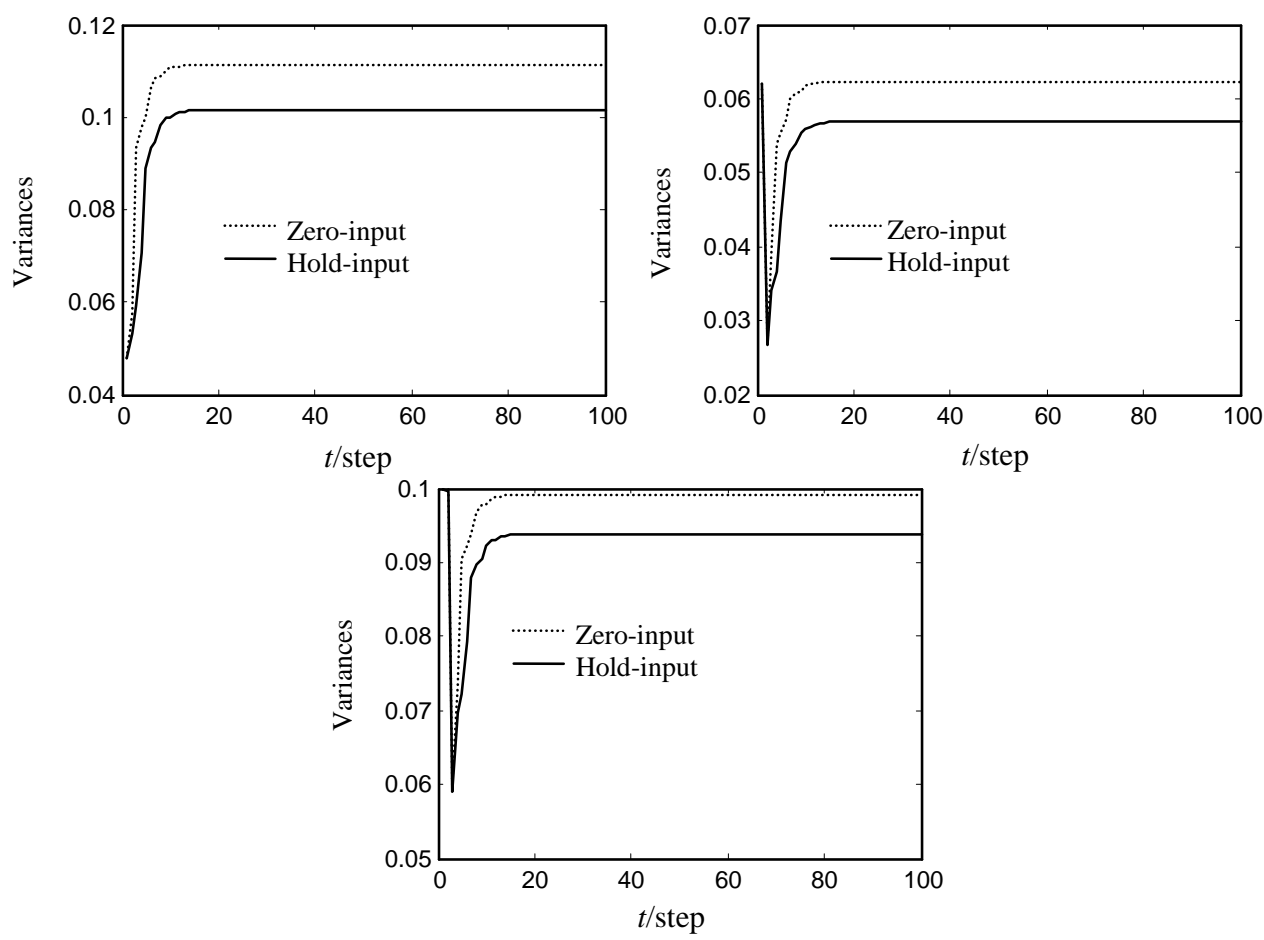

Figure 8. Filtering Error Variances under the Two Compensation Mechanisms with $\bar{\eta}=0.9$ 


\section{Conclusion}

In this article, the compensation problem of the optimal linear filter for system with random packet dropouts is investigated. The hold-input and the zero-input compensators are used to design the filters in the linear minimum variance sense, respectively. The comparison of the filtering performance of the two filters is given in simulation example section. It can be concluded from the simulation that they are all suboptimal and neither of the two compensators always outperforms the other. Hence, it is necessary to design an optimal compensator. This is also a research topic in the future.

\section{Acknowledgements}

This work is supported by the Natural Science Foundation of China (No. 61403131), Heilongjiang Province Youth Fund (QC2016092), Natural Science Foundation of Heilongjiang Province (No. F201426, No. F2015045).

\section{References}

[1] P. Antsaklis and J. Baillieul, "Special Issue on Technology of Networked Control Systems", Proceedings of the IEEE, vol. 95, no. 1, (2007), pp. 5-8.

[2] J. P. Hespanha, P. Naghshtabrizi and Y. G. Xu, "A Survey of Recent Results in Networked Control Systems", Proceedings of the IEEE, vol. 95, no. 1, (2007), pp. 138-162.

[3] L. Schenato, B. Sinopoli, M. Franceshetti, K. Poolla and S. S. Sanstry, "Foundations of Control and Estimation Over Lossy Networks", Proceedings of the IEEE, vol. 95, no. 1, (2007), pp. 163-187.

[4] K. Y. You and L. H. Xie, "Survey of Recent Progress in Networked Control Systems", Acta Automatica Sinica, vol. 39, no. 2, (2013), pp. 101-118.

[5] J. Yu, L. Wang and M. Yu, "Switched System Approach to Stabilization of Networked Control Systems", International Journal of Robust and Nonlinear Control, vol. 21, (2011), 1925-1946.

[6] M. Sahebsara, T. W. Chen and S. L. Shah, "Optimal $H_{2}$ Filtering in Networked Control Systems with Multiple Packet Dropouts”, IEEE Trans. on Automatic Control, vol. 52, no. 8, (2007), pp. 1508-1513.

[7] S. L. Sun, L. H. Xie, W. D. Xiao and Y. C. Soh, "Optimal Linear Estimation for Systems with Multiple Packet Dropouts", Automatica, vol. 44, no. 5, (2008), pp. 1333-1342.

[8] Y. Liang, T. W. Chen and Q. Pan, "Optimal Linear State Estimator with Multiple Packet Dropouts", IEEE Trans. on Automatic Control, vol. 55, no. 6, (2010), pp. 1428-1433.

[9] S. L. Sun, "Optimal Linear Filters for Discrete-Time Systems With Randomly Delayed and Lost Measurements With/Without Time Stamps", IEEE Trans. on Automatic Control, vol. 58, vol. 6, (2013). pp. 1551-1556.

[10] J. Bae, W. Zhang and M. Tomizuka, "Compensation of Packet Loss for a Network-based Rehabilitation System”, Proceedings of International Conference on Robotics Automatic, (2012), pp. 2413-2418.

[11] F. Han, G. Feng, Y. Wang, Y. Wang, J. B. Qiu, and C. Z. Zhang, "A Novel Dropout Compensation Scheme for Control of Networked T-S Fuzzy Dynamic Systems”, Fuzzy Sets System, vol. 235, (2014), 44-61.

[12] O. C. Imer, S. Yüksel and T. Başar, "Optimal Control of LTI Systems Over Unreliable Communication Links", Automatica, vol. 42, no. 9, (2006), pp. 1429-1440.

[13] L. Schenato, "To Zero or To Hold Control Inputs With Lossy Links?", IEEE Trans. on Automatic Control, vol. 54, no. 5, (2009), pp. 1093-1099.

[14] J. Gao, H. Wu and M. Fu, "Two Schemes of Data Dropout Compensation for LQG Control of Networked Control Systems", Asian Journal of Control, vol. 17, no. 1, (2015), pp. 55-63.

[15] E. I. Silva, F. J. Vargas and A. I. Maass, "Optimal Design of a Class of Controllers and Data-dropout Compensators for LTI Plants Controlled Over Erasure Channels", Proceedings of IEEE Conference on Decision and Control, (2013), pp. 6741-6746.

[16] Y. Wang, Q. Han and X. Yu, "One Step Prediction Based Packet Dropout Compensation for Networked Control Systems", American Control Conference, (2011), pp. 2849-2854.

[17] P. Shi, X. L. Luan and F. Liu, " $H_{\infty}$ Filtering for Discrete-time Systems With Stochastic Incomplete Measurement and Mixed Delays", IEEE Trans. on Industrial Electronics, vol. 59, no. 6, (2012), pp. 2732-2739. 


\section{Authors}

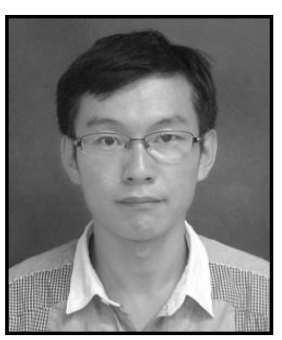

Hao Jin, he was born in Heilongjiang, China, in 1981. He received the B.E. and M.E. degrees from Heilongjiang University, Harbin, China, in 2004 and 2007, respectively. His current research interests include information fusion filtering and image processing.

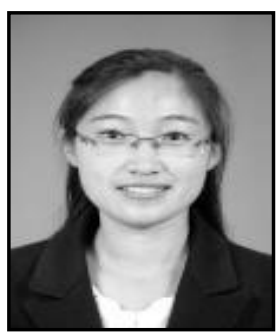

Jing Ma, she was born in Heilongjiang, China, in 1979. She received the M.E. and Ph.D. degrees from Heilongjiang University, Harbin, China, in 2007 and 2012, respectively. Her current research interests include sensor network and information fusion filtering. 
International Journal of Future Generation Communication and Networking Vol. 9, No. 11 (2016) 